EPJ Web of Conferences 71, 00019 (2014)

DOI: $10.1051 /$ epjconf / 20147100019

(C) Owned by the authors, published by EDP Sciences, 2014

\title{
Forward physics with the LHCf experiment: a LHC contribution to cosmic-ray physics
}

L. Bonechi ${ }^{1, a}$ O. Adriani ${ }^{1,2}$, E. Berti ${ }^{2}$, M. Bongi ${ }^{1,2}$, G. Castellini ${ }^{3}$, R. D’Alessandro ${ }^{1,2}$, M. Del Prete $^{1,2}$, M. Haguenauer ${ }^{4}$, Y. Itow ${ }^{5,6}, \mathrm{~K}$. Kasahara ${ }^{7}$, Y. Makino ${ }^{5}$, K. Masuda ${ }^{5}$, E. Matsubayashi ${ }^{5}, \mathrm{H}$. Menjo $^{8}$, G. Mitsuka ${ }^{2,5}$, Y. Muraki ${ }^{5}$, P. Papini ${ }^{1}$, A-L. Perrot ${ }^{9}$, S. Ricciarini ${ }^{1,3}$, T. Sako ${ }^{5}$, N. Sakurai ${ }^{6}$,

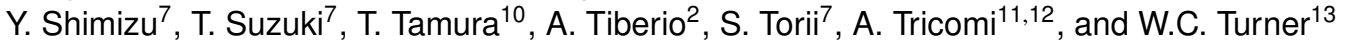

${ }^{1}$ INFN Section of Florence, Italy

${ }^{2}$ University of Florence, Italy

${ }^{3}$ IFAC-CNR, Italy

${ }^{4}$ Ecole-Polytechnique, France

${ }^{5}$ Solar-Terrestrial Environment Laboratory, Nagoya University, Japan

${ }^{6}$ Kobayashi-Maskawa Institute for the Origin of Particles and the Universe, Nagoya University,Japan

${ }^{7}$ RISE, Waseda University, Japan

${ }^{8}$ Graduate school of Science, Nagoya University, Japan

${ }^{9} \mathrm{CERN}$, Switzerland

${ }^{10}$ Kanagawa University, Japan

${ }^{11}$ INFN Section of Catania, Italy

${ }^{12}$ University of Catania, Italy

${ }^{13}$ LBNL, Berkeley, USA

\begin{abstract}
LHCf is a small detector installed at LHC accelerator to measure neutral particle flow in the forward direction of proton - proton ( $\mathrm{p}-\mathrm{p})$ and proton-nucleus $(\mathrm{p}-\mathrm{A})$ interactions. Thanks to the optimal performance that has characterized the last years' running of the LHC collider, several measurements have been taken since 2009 in different running conditions. After data taking for $\mathrm{p}$ - $\mathrm{p}$ interactions at $\sqrt{s}=900 \mathrm{GeV}, 2.76 \mathrm{TeV}$ and $7 \mathrm{TeV}$ and proton - Lead nucleus $\left(\mathrm{p}-\mathrm{Pb}\right.$ ) at $\sqrt{s_{N N}}=5.02 \mathrm{TeV}$ (energy of a couple of projectile and target nucleons in their center of mass reference frame), LHCf is now going to complete its physics program with the $13 \mathrm{TeV} \mathrm{p}$ - $\mathrm{p}$ run foreseen in 2015 . The complete set of results will become a reference data set of forward physics for the calibration and tuning of the hadronic interaction models currently used for the simulation of the atmospheric showers induced by very high energy cosmic rays. For this reason we think that LHCf is giving an important contribution for the study of cosmic rays at the highest energies. In this paper the experiment, the published results and the current status are reviewed.
\end{abstract}

\section{Introduction}

Forward physics at particle accelerators is one of the key tools that allows a clear connection between accelerator physics and cosmic - ray physics. In general these two fields of Physics cover different

\footnotetext{
a e-mail: Lorenzo.Bonechi@fi.infn.it
} 
aspects of the study of the subnuclear world, the former addressing the study of the forces between the elementary constituents of matter and of the existence of new particles, the latter investigating the relationship between elementary particles and nuclei and the astrophysical universe. The study of the charged cosmic radiation at the highest energies requires an indirect estimate of the kinematic parameters and the species of the primary particles, based on the measurements of the secondary particle and radiation cascades produced in the atmosphere, the so-called atmospheric showers. The study of the development of these cascades is crucial for the complete reconstruction of the primary events by which they are initiated. In this framework some important aspects are strictly related to the forward physics at accelerator. This is mainly due to the fact that the greatest contribution to the energy flow in an atmospheric shower is given by high energy particles produced in the first interaction and emitted in a very narrow cone along the interaction line. All the hadronic interaction models that were used in the study of atmospheric showers before the LHCf data were available, were based necessarily on a rough description of the fluxes and energy spectra of forward particles at very high energy, because only a few measurements existed of forward particle spectra at high energy [1,2]. The uncertainties on the hadronic interaction models determine necessarily a systematic uncertainty in the reconstruction of the primary cosmic - ray events. As a result it is not possible yet to give an answer to some open problems in cosmic - ray physics, like the composition of cosmic rays at the knee and beyond or the existence of the GZK cutoff at extreme energies.

\section{The LHCf experiment}

Apart from $\mathrm{LHCb}$, all the other big detectors at the LHC cover simmetrically the central region of the $\mathrm{p}$ - $\mathrm{p}$ interaction, i.e. the region at high angles with respect to the interaction line, within a pseudorapidity range that can extend up to about 2.5 in absolute value, if we consider the inner tracking subdetectors, or up to 5 if we consider only the calorimetric parts. In case of LHCb the apparatus is asymmetric with respect to the interaction point and the pseudo rapidity coverage is between 2 and 5 . The reason of the impossibility to access the more forward region for these experiments is the presence of the vacuum tube around the beam line (the beam pipe). Particles emitted in the forward direction travel inside this tube and cannot be detected by any of these apparatus. After approximately $140 \mathrm{~m}$ from the ATLAS interaction point, IP1, on both left and right sides, the beam pipe enter the so-called recombination chambers, where the single tube coming from IP1 turns in a couple of smaller tubes with a $96 \mathrm{~mm}$ wide gap between them. These regions are surrounded by the big massive structures called TANs, that are used to protect the downstream cryogenic devices from neutral particle debris produced in the interactions (figure 1). Only neutral debris can in fact enter the TAN regions, because charged ones are deflected by the dipole magnet located between IP1 and the TANs themselves. The TAN structure is such as to allow the insertion of thin detectors directly on the the beam line, that is between the two small pipes. A $60 \mathrm{~cm}$ high, $96 \mathrm{~mm}$ large and $1 \mathrm{~m}$ long slot is in fact accessible from the top of the structure to be instrumented with radiation detectors. Inside the recombination chamber the vacuum pipe is machined in such a way that there is a $10 \mathrm{~cm} \times 10 \mathrm{~cm}$ windows centered on the beam axis from which the projection of its thickness up to IP1 amounts precisely to one radiation length.

The LHCf detectors occupy the first $30 \mathrm{~cm}$ of the TAN slots, facing the interaction point, thus minimizing the material between them and IP1. This is the best condition allowed at the LHC for measuring fluxes and energy spectra of high energy forward neutral particles produced by the interacting beams. This is the main purpose of the LHCf experiment [3]. 

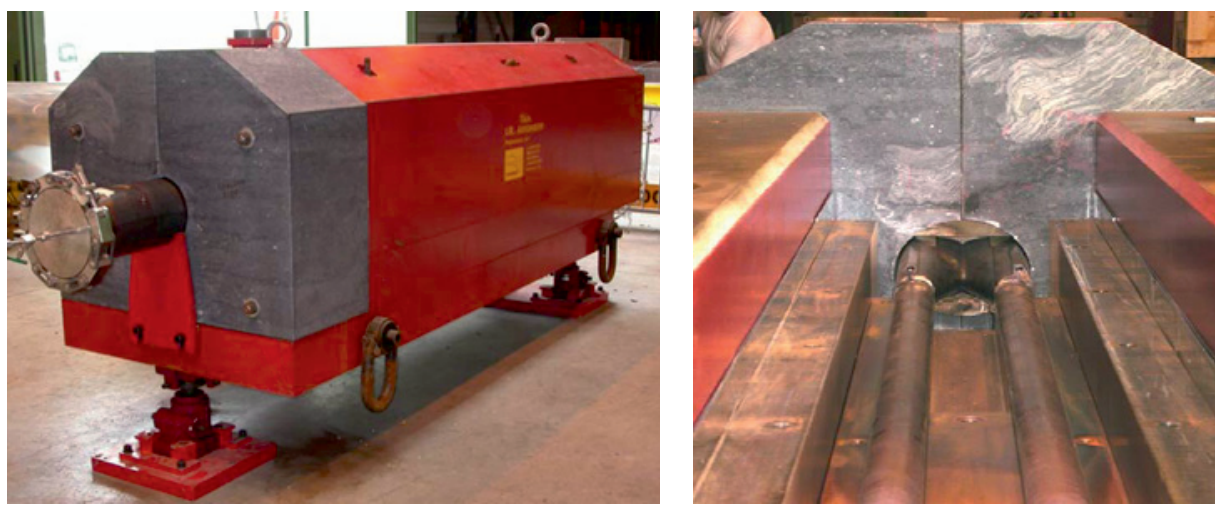

Figure 1. Left: the massive absorber (TAN) used to stop neutral particles produced in the forward direction in the beam interactions; on the left side the flange for the connection to the beam pipe on IP1 side can be easily identified. Right: the recombination chamber inside the TAN absorber; in this volume the single beam pipe coming from IP1 turns into two smaller diameters tubes, $9.6 \mathrm{~cm}$ far from each other, that can be identified in the lower central part of the picture.

\subsection{The detectors}

Both LHCf detectors [4] are made of a couple of $44 X_{0}$ deep sampling electromagnetic calorimeters, usually called "towers", with an impact surface of a few $\mathrm{cm}^{2}$. A single tower consists of squaresection tungsten and scintillator tiles, used as absorber and sampling layers respectively. In spite of the small transverse size of the towers, comparable with the Molière radius of Tungsten $\left(R_{M} \sim 9 \mathrm{~mm}\right)$, the high longitudinal granularity results in a good energy resolution for electromagnetic showers and gives the possibility of doing a detailed study of the longitudinal development of the energy release that is helpful for particle identification. The transverse development, particularly important for determining the particle's impact point and its distance from the calorimeter edge (to reject events with excessive energy leakage outside the calorimeter), is studied by means of four X - Y tracking layers made of $1 \mathrm{~mm}$ x $1 \mathrm{~mm}$ square cross section scintillating fibers for detector I (called Arm1) and $160 \mu \mathrm{m}$ readout pitch micro - strip silicon sensors for detector II (called Arm2) [3-5]. The separation of a single detector into two separate and almost independent calorimeters is required to minimize the overlap of signals due to the different simultaneous showers when detecting for example the two gamma rays produced in a neutral pion decay. The different geometries of the two detectors [4] have been carefully studied to maximize the accessible kinematic region and exploit the different characteristics of the position sensitive detectors.

\section{Published results}

Until now the LHCf experiment has taken data during two different runs of the LHC. The former in 2010, when $\mathrm{p}$ - $\mathrm{p}$ interactions at $\sqrt{s}=900 \mathrm{GeV}$ and $\sqrt{s}=7 \mathrm{TeV}$ were produced. The latter in 2013 when the interacting particles were protons and Lead nuclei at $\sqrt{s_{N N}}=5.02 \mathrm{TeV}$. At the end of the last run $\mathrm{p}$ - $\mathrm{p}$ interactions at $\sqrt{s}=2.76 \mathrm{TeV}$ were also produced for a short time. The already published results for the $\mathrm{p}-\mathrm{p}$ runs are collected in references [6-8]. The main results for the higher energy run are the measurements of the single $\gamma$-ray energy spectrum in two different pseudo - rapidity ranges and the study of the transverse momentum spectra of the $\pi^{0}$ component. As a general result, the model 
that better reproduces the LHCf data is EPOS 1.99. Large discrepancies are found especially for the other models under investigation. The experimental data lie between expectations by the different models, yet the data quality is such as to allow an improvement of the models' predictions, once taken properly into account. Model deviations of models from data have also been observed for data taken at $900 \mathrm{GeV}$.

\section{The proton - Lead nucleus run}

After data taking in 2010 for the $7 \mathrm{TeV}$ p - p run at the LHC, the LHCf detector were uninstalled from their locations, allowing the ATLAS ZDC electromagnetic modules to be installed in the same locations, on both sides of IP1. Both LHCf detectors were brought outside the tunnel for testing and upgrading. Between the end of 2012 and January 2013 one of the two LHCf detectors was installed again in the LHC tunnel for taking data during the $\mathrm{p}-\mathrm{Pb}$ run at $\sqrt{s_{N N}}=5.02 \mathrm{TeV}$. The selected detector was Arm2, that has the best performance in terms of spatial resolution. It was re-installed before being upgraded, in the original configuration. The reasons for requiring the installation of only one detector were different. Firstly the minimization of the interference with the ZDC physics plan, but also the result of our simulations, showing that the most feasible measurement for the LHCf detector was on the proton remnant side of the $\mathrm{p}-\mathrm{Pb}$ interaction, due to the high neutron multiplicity on the other side. The plan defined by the LHC coordination team was to keep the beam configuration fixed for almost the total time of the run and to swap the beams near the end of the run for a short time. Therefore it was decided to install only one detector on the proton remnant side, assuming that LHCf would have the possibility to take data for limited time also on the Lead remnant side (after the swap of the beams).

The data collected during the period between January 18 and February 14, 2013 is approximately of $2 \times 10^{8}$ events on the proton remnant side and of $5.6 \times 10^{6}$ on the Lead remnant side. Especially in the former case, different configurations have been used during data taking, both for detector calibration and for physics. The whole data are therefore divided into different sets. In particular different vertical positions of the detector were used in such a way to extend the accessible phase space within the limits of the beam pipe geometry.

A first limited set of data has been selected for the preliminary analysis presented in this paper.

\subsection{Preliminary analysis: data selection}

The selected data set contains $1.23 \times 10^{6}$ triggers taken on February 01, between 05:46 and 06:15, during the LHC fill number 3510. The configuration of the beam was the nominal one defined for the $\mathrm{p}-\mathrm{Pb}$ run, with 338 bunches, $290 \mu \mathrm{rad}$ beam crossing angle and $\beta^{*}=0.81 \mathrm{~m}$. The LHCf detector was on the proton - remnant side of the interaction. The detector's configuration was the nominal one, with the smaller calorimeter aligned with the beam line (two centimeters lower than the neutral beam line due to the non-zero beam crossing angle). As reported on the LHC official tables [9], during fill number 3510, the beams were declared as stable at 04:26 and lasted for 16012 seconds after this time. The initial (peak) luminosity was $L_{0}=98.780 \times 10^{27} \mathrm{~cm}^{-2} \mathrm{~s}^{-1}$ and the mean lifetime of the luminosity was $\tau_{i}=18761 \mathrm{~s}$ at the beginning of the stable beams and $\tau_{f}=24605 \mathrm{~s}$ at the end. The uncertainty on the luminosity is roughly estimated to be around $20 \%$. The start of data taking for the selected set was after $2580 \mathrm{~s}$ after the stable - beams were declared and lasted $1785 \mathrm{~s}$. The total acquisition live time, determined thanks to a counter variable written to the data file for each event, is $51.4 \mathrm{~s}$. Considering these values and taking into account the uncertainty on the luminosity and the total inelastic cross section $\sigma_{\text {ine }}=(2.09 \pm 0.12) \mathrm{b}$ [10], the mean luminosity is estimated as $L_{\text {mean }}=$ 

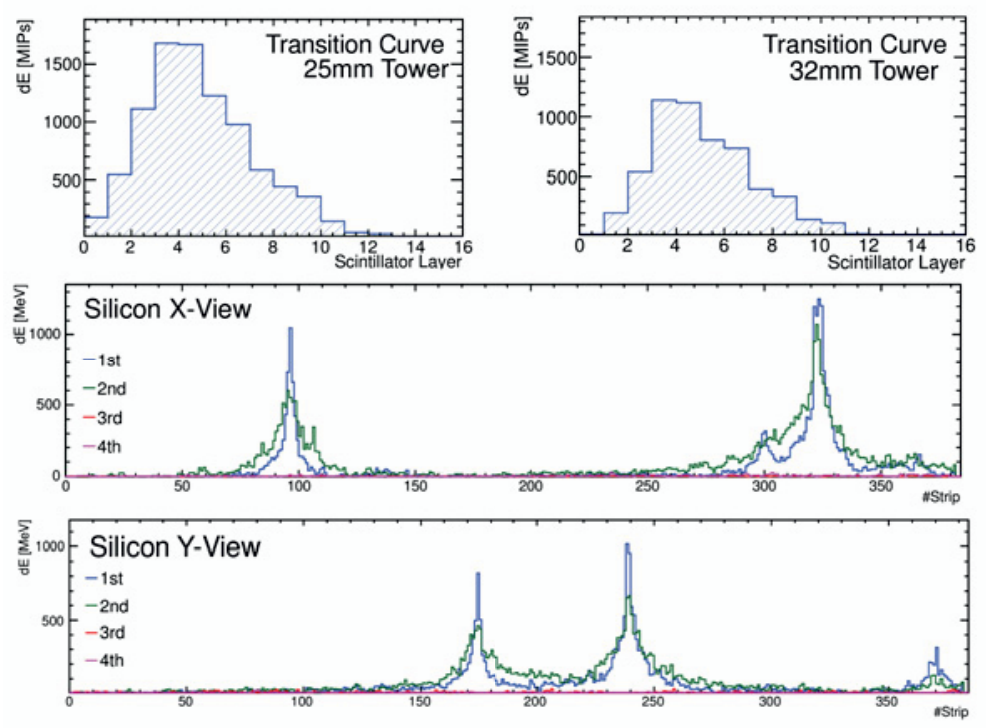

Figure 2. Summary plots for a multiple hit event. Upper two plots: longitudinal transition curves measured by means of the scintillator tiles on the two calorimeter towers. Lower plots: transverse shape of the detected showers measured by means of the four $\mu$-strip silicon detector planes.

$(8.3 \pm 1.7) \times 10^{28} \mathrm{~cm}^{-2} \mathrm{~s}^{-1}$ and the total number of inelastic collisions results $N_{\text {ine }}=(0.89 \pm 0.19) \times 10^{7}$. In these calculations it is assumed that the luminosity lifetime varies linearly with time after that stable beams are declared.

\subsection{The electromagnetic component: photons}

The e.m. component (photons) is the most precisely measured by LHCf, since the detector is optimized just the reconstruction of e.m. showers. The method for the measurement of the impact points and for the calibration of the energy measurement are described in [6]. Only single particle events have been selected for this preliminary analysis and only statistical uncertainties are considered in the plots. Events with multiple hits inside the calorimeters are rejected thanks to the information given by the silicon $\mu$-strip modules. In figure 2 the longitudinal transition curves for the two calorimeter towers, measured by the scintillating system, and the transverse distribution of the energy released in the silicon detectors, are shown for one event. This event is clearly affected by multiple hits that can be easily identified in the lower plots. The hadronic events, due to their higher penetration power with respect to the e.m. ones, are identified by studying the longitudinal shower development thanks to the good calorimeter's depth $\left(44 X_{0} \simeq 1.55 \lambda_{\text {int }}\right.$ ). To maximize the selection performance and the purity of the identified samples of particles, the selection cuts depend on the total energy release measured inside the calorimeter. The determination of an incoming particle's energy is obtained by summing the energy releases measured in all the scintillator tiles, independently for each calorimeter tower, and applying an appropriate conversion function. The uncertainty on the energy determination for e.m. events is better than $5 \%$ above $100 \mathrm{GeV}$.

A drift of the measured value of the neutral pion mass was found during the whole data taking period. Neutral pions can be identified for example among the events with one e.m. shower in each tower. The 

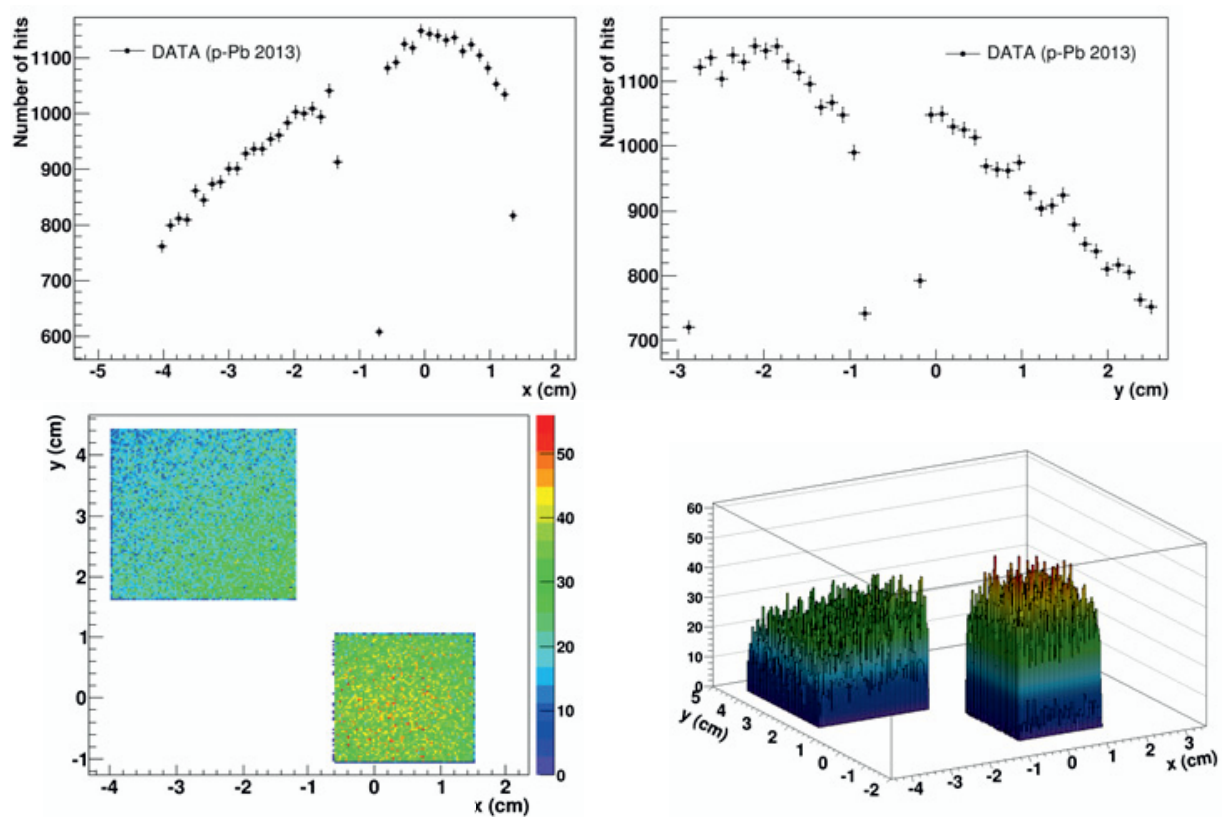

Figure 3. Upper figures: distributions of photon impact points along the $\mathrm{x}$ and $\mathrm{y}$ coordinates, transverse to the beam line ( $\mathrm{x}$-axis is horizontal and $\mathrm{y}$-axis is vertical); the origin in these plots is chosen as the neutral LHC beam line, while, due to the $290 \mu \mathrm{rad}$ beam crossing angle, the detector was positioned $2 \mathrm{~cm}$ lower than the default position. Lower figures: two-dimensional photon impact-point maps; in this plots the origin of the reference frame is coincident with the real beam center (determined as discussed in section 4.3).

reconstructed $\pi^{0}$ mass changes during the run mostly due to temperature drift and radiation damage. This was taken into account by correcting the energy reconstruction routines accordingly.

Further details about the methods used for the analysis are described in [6].

In figure 3 the distribution of photon impact points is shown. From the first two plots, where the impact point distribution is shown separately along the $\mathrm{x}$ and $\mathrm{y}$ directions, transverse to the beam line, it is easy to separate the contributions from the two calorimeter towers: the small one is located on the beam line and has a higher flux than the big one. The two dimensional impact point map is shown in the last plot. From this study it can be seen that the photon flux is spread out around the beam line and does not allow a precise identification of the beam center.

The small tower includes events with pseudo - rapidity greater than 9.6 while the large tower includes events with pseudo - rapidity between 8.4 and 9.4. The energy spectrum of the photon component has been preliminary evaluated separately for the two complete calorimeter towers, without identification of precise intervals of the pseudo rapidity. The results of this preliminary analysis is shown in figure 4. The spectra reported in this figure are normalized to the number of inelastic interactions evaluated as described in section 4.1.

In figure 5 the invariant mass distribution for events containing one single e.m. shower for each tower is shown. The invariant mass is obtained assuming that the couple of photons come from IP1. From this histogram the peak due to the $\pi^{0}$ - decay photons is clearly visible at $W \simeq 135 \mathrm{MeV}$.

A detailed comparison of the LHCf data with the predictions from the available hadronic interaction models is ongoing and will be the subject of a future publication. 


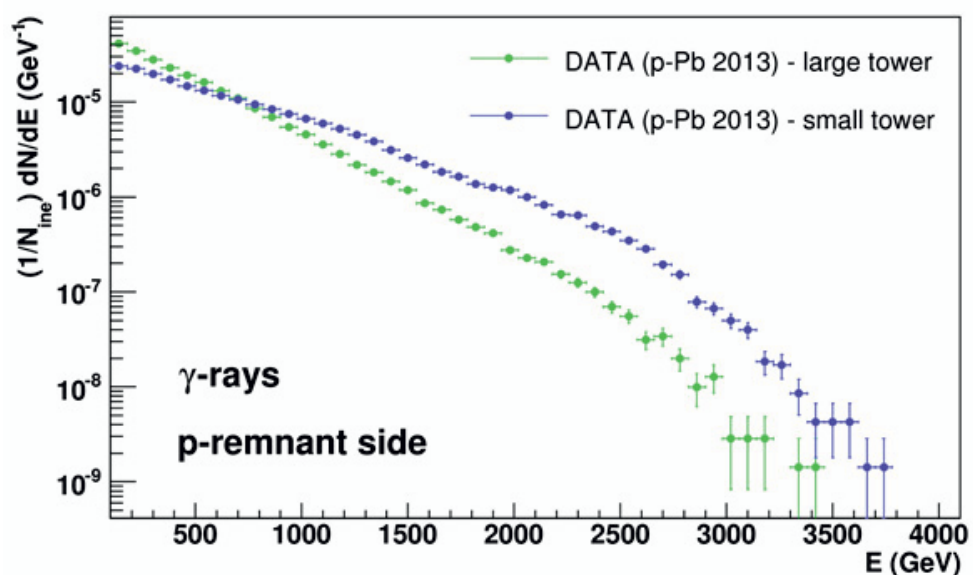

Figure 4. Energy spectrum for single photon events measured separately by the two calorimeter towers of the Arm 2 detector. The spectrum on the small calorimeter tower, aligned with the beam line, is harder that the spectrum on the other tower. The vertical error bars are the statistical uncertainties.

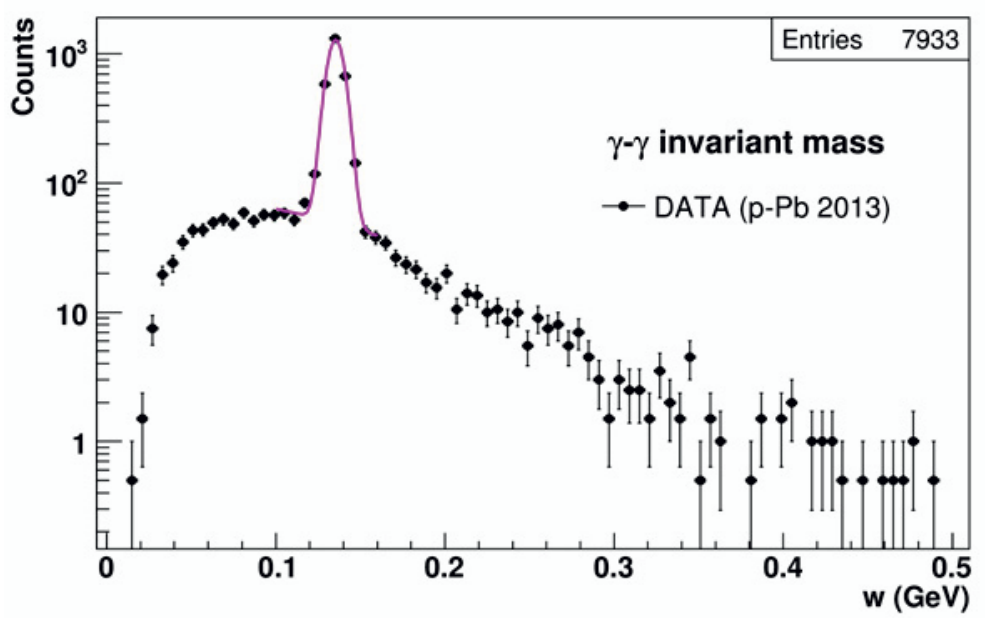

Figure 5. Invariant mass of couple of $\gamma$ - rays entering separately the two calorimeter towers of the Arm 2 detector. The $\pi^{0}$ mass peak can be easily identified. A selection on the invariant mass for $\pi^{0}$ events can be applied with a contamination around $9 \%$ from the background.

\subsection{The hadronic component: neutrons}

The study of the hadronic component of the neutral particle flux in LHCf is more complicated and less precise with respect to the e.m. case. This is mainly due to the detector's dimensions both in the transverse and in the longitudinal directions, which do not allow full containment of the showers. Nonetheless important information on the hadronic event spatial distribution and energy spectra can 

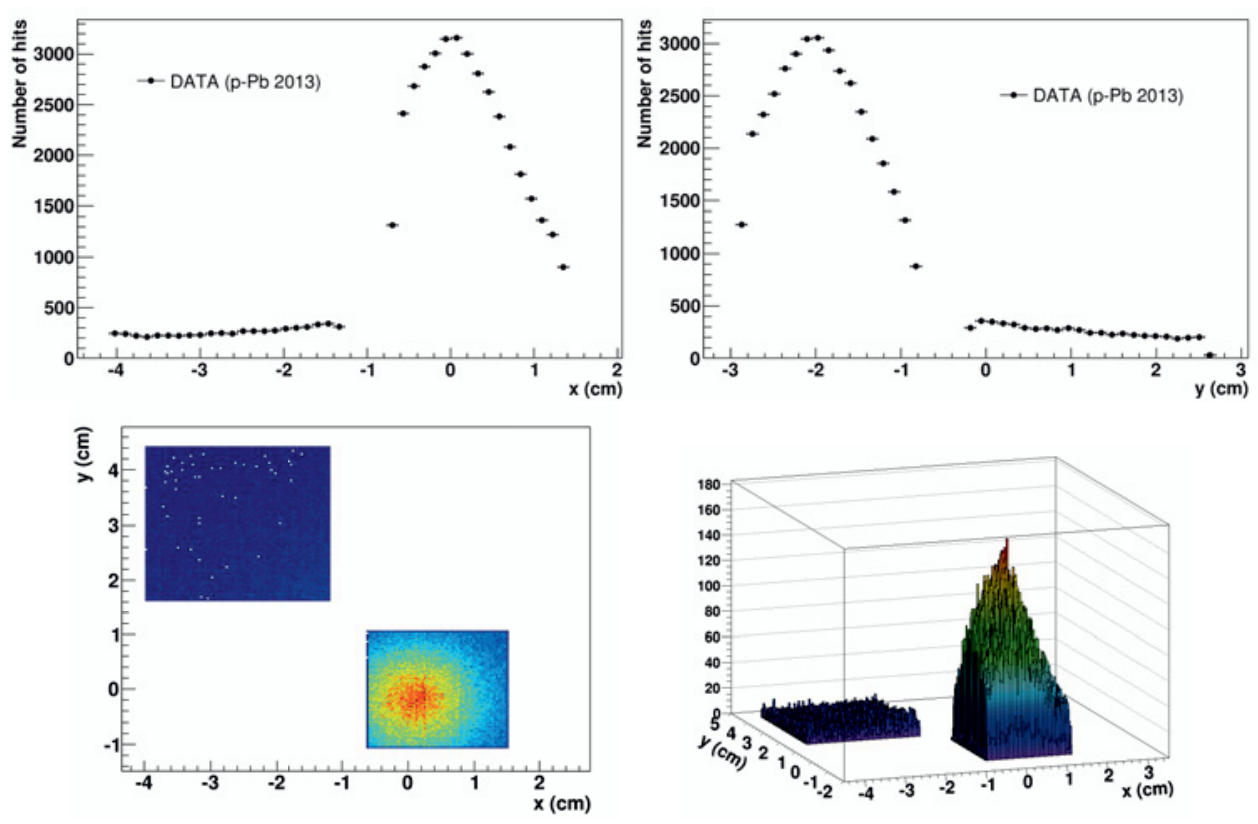

Figure 6. Upper figures: distributions of single hadron impact points along the $\mathrm{x}$ and $\mathrm{y}$ coordinates, transverse to the beam line (x-axis is horizontal and $\mathrm{y}$-axis is vertical); the origin in these plots is chosen as the neutral LHC beam line, while, due to the $290 \mu \mathrm{rad}$ beam crossing angle, the detector was positioned $2 \mathrm{~cm}$ lower than the default position. Lower figures: two and three - dimensional hadron impact point maps; in this plots the origin of the reference frame is coincident with the real beam center (determined as discussed in the text). The peak of the hadron flux in correspondence of the small calorimeter tower can be easily identified in the three - dimensional histogram (bottom right plot).

be recovered. In figure 6 the distribution of the impact points of particles that are identified as hadrons, mainly neutrons, is shown.

It can be clearly seen that the hadron flux is much more peaked in the forward direction than the gamma - ray flux. The hit map reconstructed by LHCf allows a precise determination of the beam center. Its measured coordinates with respect to the nominal position are $x_{B C}=(1.07 \pm 0.09) \mathrm{mm}$ and $y_{B C}=(-1.87 \pm 0.08) \mathrm{mm}$. The behavior of the neutral hadron flux at very high rapidity is under investigation and a comparison with the expectations by the available hadronic interaction models will be published soon.

In figure 7 the energy distribution of the hadronic component, measured by the small and large tower separately, are shown. It should be considered that the algorithm used for the energy reconstruction of hadronic particles is currently under development. The horizontal axis does not represents in fact the real energy reconstructed for the incoming hadron, but the hadron $\gamma$-ray - equivalent energy, that is the reconstructed energy of the incoming particle on the basis of the total energy released in the hit tower, assuming that it is a $\gamma$ - ray particle. This procedure does not yet take into account the detector's response for the hadron component $[11,12]$. A complete unfolding procedure is currently under test for the analysis of the $\sqrt{s}=7 \mathrm{TeV}$ data. 


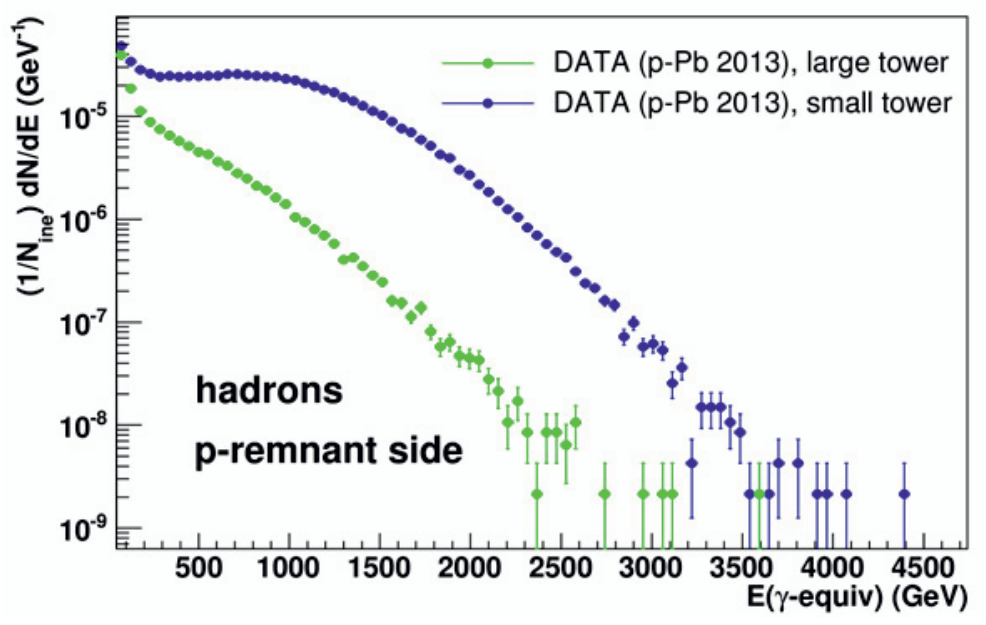

Figure 7. Energy spectrum for single hadron events measured separately by the two calorimeter towers of the Arm 2 detector. As for the $\gamma$ - ray spectra (figure 4) the spectrum on the small calorimeter tower, aligned with the beam line, is harder that the spectrum on the other tower, as expected. The vertical error bars are the statistical uncertainties.

\section{Current status and ongoing analysis}

Currently several analyses are ongoing and first results will be published probably during 2014. The main point concerning the $\mathrm{p}$ - $\mathrm{p}$ data taken in 2010, after the results for single $\gamma$-rays and $\pi^{0}$, is the study of the hadron component. The algorithm for the reconstruction of the real neutron spectrum is not yet completely defined, with the procedure for spectrum unfolding still under test. At the same time the analysis of the $\mathrm{p}-\mathrm{Pb}$ data collected in 2013 is also underway, but the study of all the $\gamma$-ray, $\pi^{0}$ and hadron components have still to be completed. The nuclear effects of hadronic interactions that can be studied in the forward region by comparing the results for the $\mathrm{p}-\mathrm{Pb}$ run with those for the $\mathrm{p}$ - $\mathrm{p}$ runs, can have an important impact on the calibration of the hadronic interaction models for cosmic - ray studies. A third part of the analysis is dedicated to the beam test data that have been collected at the SPS accelerator at CERN for detector calibration. In particular a detailed study of the temperature dependence of the detectors' response is under investigation in order to correct for the drift of the $\pi^{0}$ mass peak that has been observed in the 2010 and 2013 data.

In parallel to data analysis, the LHCf collaboration is now involved in two different problems. One is the upgrade of both the detectors for the $\mathrm{p}-\mathrm{p}$ run at $\sqrt{s}=13 \mathrm{TeV}$ at LHC, foreseen for 2015 . All the plastic scintillator components have been replaced with GSO for better resistance to radiation damage and the new scintillator systems are now under test. In addition the silicon planes of the Arm2 detector will be completely renewed to reduce the electronics saturation effect for showers induced by high energy $(\mathrm{TeV})$ particles and correct a minor fault on the front - end circuitry. The second activity concerns a possible future run of the LHCf detectors at the RHIC facility at the Brookhaven National Laboratory (BNL). This activity takes the name of RHICf [13]. The RHIC accelerator provides $\mathrm{p}-\mathrm{p}$ interactions at lower energy than LHC, but due to the different location along the beam line, it accesses a region of the $p_{t} / x_{F}$ (where $p_{t}$ is the transverse momentum and $x_{F}$ the Feynman $\mathrm{x}$ variable) phase space that is comparable to the region allowed at the LHC for the $7 \mathrm{TeV} \mathrm{p}$ - $\mathrm{p}$ case. This is important for comparing the results at different energies and to give an experimental basis to the Feynman scaling, 
which is of interest for the extrapolation of the LHC results at much higher energies, required for the study of the extreme energy cosmic - ray particles. Moreover a proton - light ion run, extremely interesting because of its relevance to the cosmic - ray interactions in atmosphere, is being considered both at LHC and at RHIC.

\section{Conclusions}

The purpose of this work is a general presentation of the LHCf experiment at the LHC [3-5]. The aim of this experiment, composed by two separate detectors installed along the LHC beam line at $\pm 140 \mathrm{~m}$ from the ATLAS Interaction Point, is the study of the neutral particle flux in the forward region of the $\mathrm{p}-\mathrm{p}$ or $\mathrm{p}$ - A interaction at high energy. This kind of measurements are necessary for the calibration of the hadronic interaction models used in cosmic - ray physics for the study of the atmospheric showers. LHCf begun its data taking in 2009 for $\mathrm{p}$ - $\mathrm{p}$ interactions at $\sqrt{s}=900 \mathrm{GeV}$ and $\sqrt{s}=7 \mathrm{TeV}$. Interesting results for single $\gamma$ - ray and $\pi^{0}$ have been already published [6-8] and a study of the neutron component is at an advanced stage for publication. In 2013 one of the two detectors has taken data also during the $\mathrm{p}-\mathrm{Pb}$ run at $\sqrt{s_{N N}}=5.02 \mathrm{TeV}$. The analysis of these new data is also ongoing. Results for both the analysis will be published in 2014. The main other works in progress for the future activities are the complete upgrade of both the detectors for the $\sqrt{s}=13 \mathrm{TeV} p$ - $\mathrm{p}$ run on 2015 and the preparation of a new run at the RHIC facility. These activities conclude the main part of the LHCf physics program, as proposed in the LOI of the experiment, dated in 2004.

\section{References}

[1] W. Flauger, F. Mönnig, Nucl. Phys. B 109 (1976) 347

[2] E. Pare et al., Phys. Lett. B 242 (1990) 531

[3] LHCf Technical Design Report, CERN-LHCC-2006-004

[4] O. Adriani et al., JINST 3 (2008) S08006

[5] O. Adriani et al., JINST 5 (2010) P01012

[6] O. Adriani et al., Physics Letters B 703 (2011) 128-134

[7] O. Adriani et al., Physics Letters B 715 (2012) 298-303

[8] O. Adriani et al., Physical Review D 86, 092001 (2012)

[9] https://lhc-statistics.web.cern.ch/LHC-Statistics/index.php

[10] The LHCb Collaboration, LHCb-CONF-2012-034, 15/01/2013

[11] Kawade et al., submitted to JINST, 2014

[12] K. Kawade et al., Proc. 33rd ICRC, Rio de Janeiro 2013

[13] arXiv:1401.1004 [physics.ins-det] 